\title{
Quantifying Myocardial Strain of the Left Ventricle in Normal People Using Feature-Tracking Based on Computed Tomography Imaging
}

\author{
Na Li ${ }^{1,2,+}$, Tong Liu 1,2,+, Jia Liu 1,2, Yukun Cao ${ }^{1,2}$, Yumin Li ${ }^{1,2}$, Jie Yu 1,2, Xiaoyu Han ${ }^{1,2}$, Guozhu Shao ${ }^{1,2}$, \\ Ming Yang ${ }^{1,2}$, Zhihan $\mathrm{Xu}^{3}{ }^{\mathbb{D}}$, Wenjuan Zeng ${ }^{4, *}$ and Heshui Shi ${ }^{1,2, * \mathbb{D}}$
}

check for

updates

Citation: Li, N.; Liu, T.; Liu, J.;

Cao, Y.; Li, Y.; Yu, J.; Han, X.; Shao, G.; Yang, M.; Xu, Z.; et al. Quantifying

Myocardial Strain of the Left

Ventricle in Normal People Using

Feature-Tracking Based on

Computed Tomography Imaging.

Diagnostics 2022, 12, 329.

https://doi.org/10.3390/

diagnostics12020329

Academic Editor: Andrea D. Annoni

Received: 24 December 2021

Accepted: 24 January 2022

Published: 27 January 2022

Publisher's Note: MDPI stays neutral with regard to jurisdictional claims in published maps and institutional affiliations.

Copyright: (C) 2022 by the authors. Licensee MDPI, Basel, Switzerland. This article is an open access article distributed under the terms and conditions of the Creative Commons Attribution (CC BY) license (https:// creativecommons.org/licenses/by/ $4.0 /)$.
1 Department of Radiology, Union Hospital, Tongii Medical College, Huazhong University of Science and Technology, 1277 Jiefang Rd., Wuhan 430022, China; ncln1001@163.com (N.L.); Liutong_232022@163.com (T.L.); d201981614@hust.edu.cn (J.L.);yukuncao@hust.edu.cn (Y.C.); liyumin1215@163.com (Y.L.); gavinyuwhuh@hust.edu.cn (J.Y.); xiaoyuhan1123@163.com (X.H.); sgz15755376608@163.com (G.S.); ming.y@163.com (M.Y.)

2 Hubei Province Key Laboratory of Molecular Imaging, Wuhan 430022, China

3 CT Collaboration, Siemens Healthineers Ltd., Shanghai 200126, China; zhihan.xu@siemens-healthineers.com

4 Department of Clinical Laboratory, Union Hospital, Tongji Medical College, Huazhong University of Science and Technology, Wuhan 430022, China

* Correspondence: wjzengwh@163.com (W.Z.); heshuishi@hust.edu.cn (H.S.); Tel.: +86-138-7108-9008 (H.S.)

+ These authors contributed equally to this work.

\begin{abstract}
Objective: The objective was to evaluate the normal value of left ventricular myocardial strain using the computed tomography feature-tracking technique and to explore the correlation between myocardial strains and cardiac function parameters. Methods: Participants suspected of coronary heart disease were selected from 17 August 2020 to 5 November 2020 to undergo coronary computed tomography angiography using a third-generation dual-source CT scanner. Data were imported into a commercial software (Medis) after multiphase reconstruction. The cardiac function parameters, radial (Err), circumferential (Ecc), and longitudinal strain (Ell) of the left ventricle were recorded. Results: A total of 87 normal subjects were enrolled, including 41 males and 46 females. For healthy subjects, the global radial strain (GRS), circumferential strain (GCS), and longitudinal strain (GLS) of the left ventricle were $74.5 \pm 15.2 \%,-22.7 \pm 3.0 \%$, and $-26.6 \pm 3.2 \%$, respectively. The Err and Ecc absolute values $(\mid$ Ecc $\mid$ ) were the largest at the apex, and the IEll $\mid$ gradually increased from the base to the apex. The Err and IEcc| were the largest in the lateral and inferior wall, respectively. | Ell | showed a clockwise decrease from the lateral wall in the short axis. Meanwhile, the GRS and IGLSI in females were higher than that in males. Multiple linear regression analysis showed that both SV and LVEF were the independent determinants of GRS, GCS, and GLS. BMI and CO were the independent determined factors of GCS. Conclusions: At a reasonable radiation dose, CT feature-tracking is a feasible and reproducible method to analyze left ventricular myocardial strain. Left ventricular myocardial strain in normal subjects varies in gender, segments, levels, and regions.
\end{abstract}

Keywords: computed tomography imaging; feature tracking; myocardial strain

\section{Introduction}

Myocardial strain is defined as heart deformation during the systolic process, including radial strain (Err), circumferential strain (Ecc), and longitudinal strain (Ell) [1]. Featuretracking (FT) is a highly reproducible way to measure myocardial strain, evaluating global and regional myocardial strain [2]. Myocardial strain of the left ventricle is an effective indicator to predict the outcomes of cardiovascular diseases, such as ischemic cardiomyopathy and non-ischemic cardiomyopathy [3]. Common methods to evaluate the systolic function include speckle-tracking imaging ultrasound and feature-tracking based on cardiac magnetic resonance imaging (FT-CMR). However, ultrasound (US) has a poor acoustic 
and depends on the operating angle and techniques. Furthermore, the time-consuming procedure of CMR in scanning limits its usage [4]. Computed tomography imaging (CT) is a rapid, convenient way to provides a one-stop evaluation of cardiac function when assessing coronary artery anatomy and is more standardized than US. Feature-tracking based on computed tomography (FT-CT) is an excellent way to assess cardiac function and is consistent with US and CMR [5-7]. However, many studies [3,8-10] have shown normal values of the left ventricular myocardial strain by US and CMR, whereas using FT-CT to evaluate the strain of the left ventricular myocardium in healthy subjects has rarely been reported.

Therefore, this study evaluates the normal values of the left ventricular myocardium strain by FT-CT. In addition, the correlations between myocardial strain and cardiac function parameters, gender, and age are analyzed to explore the feasibility and repeatability of the application of this technique, which could provide an important reference for clinical research and applications.

\section{Materials and Methods}

\subsection{Study Population}

An ethics committee institution of Tongji Medical College of Huazhong University of Science and Technology approved the study. People suspected of coronary heart disease (CAD) underwent coronary computed tomography angiography (CCTA) from 17 August 2020 to 5 November 2020, from which normal cases without coronary artery abnormality were selected. The exclusion criteria based on history were as follows: (1) electrocardiogram (ECG) abnormalities 2 weeks before the CT examination (T wave abnormalities, ST-T changes, and atrioventricular block); (2) coronary heart disease, cardiomyopathy, valvular heart disease, and other cardiovascular diseases; (3) history of revascularization; (4) history of hypertension, diabetes, or dyslipidemia. The exclusion criteria based on cardiac CT reports were as follows: (1) coronary artery calcification score $>0$; (2) coronary artery stenosis ( $\geq 1 \%$ lumen stenosis); (3) poor cardiac CT image quality.

\subsection{Image Acquisition}

CT was performed using a Siemens third-generation dual-source CT scanner (Somatom Force, Siemens Healthineers, Forchheim, Germany). Participants were trained to inhale and hold their breath before the examination. Retrospectively, the ECG-gated coronary computed tomography angiography (CCTA) scanning parameters were as follows: detector collimation $192 \times 0.6 \mathrm{~mm}$, gantry rotation time $0.25 \mathrm{~s} / \mathrm{r}$, pitch 0.15 , and slice $0.75 \mathrm{~mm}$. Automatic tube voltage technology (Care kV, Siemens Healthineers, Forchheim, Germany) and intelligent tube current scanning technology (Care Dose 4D, Siemens Healthineers, Forchheim, Germany) were used to automatically determine tube voltage and current. The reference tube voltage range was $100 \mathrm{kv}$, and reference tube current was $350 \mathrm{mAs}$. According to the weight of subjects, a total of $30-60 \mathrm{~mL}$ iopromide $(400-\mathrm{mg} \mathrm{I} / \mathrm{mL}$; Bracco, Patheon Italia S. P. A, Ferentino, Italy) was injected into the median cubital vein, followed by diluted contrast and a saline flush. Diluted contrast was mixed saline solution with a ratio of 2:8, and the injection rate was $2-4 \mathrm{~mL} / \mathrm{s}$. The monitored region of interest was placed at the aortic root, and the trigger threshold of coronary artery CTA automatic scanning was set at $100 \mathrm{HU}$. When the density within the region of interest reached the threshold, scanning was automatically triggered, and the scanning time was 5-6 s. Twenty phases were reconstructed in 5\% steps of the RR interval within the full window. The data constructive section thickness was $0.75 \mathrm{~mm}$, the increment was $0.5 \mathrm{~mm}$, the reconstruction kernel was Bv40, and the model-based iterative reconstruction (ADMIRE, Siemens Healthineers, Forchheim, Germany) was at a strength level of 3. The effective radiation dose was $4.0 \pm 1.4 \mathrm{mSv}$ (range: 1.9-6.7 mSv), equal to DLP (dose-length product) multiplied by 0.014 


\subsection{Image Post-Processing and Analysis}

The 20-phase images were imported to a commercial software package (Medis suite v3.0, Leiden, The Netherlands) to analyze the myocardial strain. Based on the American Heart Association 16-segment model for the left ventricle, the left ventricular basal, middle, and apex segments were selected for strain analysis. The end-diastole and -systole of the left ventricle was determined on the short- and long-axis sections, and the endocardial and epicardial borders were manually delineated, respectively. The deformation of myocardial movement was obtained by automatically tracking the continuous contour of the endocardial and epicardial borders throughout the cardiac cycle. In addition, the parameters of cardiac function were recorded, including the left ventricular end-diastolic volume (LVEDV), left ventricular end-systolic volume (LVESV), stroke volume (SV), cardiac output (CO), left ventricular ejection fraction (LVEF), and global and regional segment myocardial strain. The anterior wall of the left ventricle included segments 1, 2, 6, 7, 8, 12, and 13 . The septal wall of the left ventricle included segments $2,3,8,9$, and 14 . The inferior wall included segments $3,4,5,9,10,11$, and 15. The lateral wall included segments 5, 6, 11, 12, and 16. The Err and Ecc were measured in the short axis of the heart, and the Ell was an average value measured in the long axis (two, three, and four chambers) of the heart (Figure 1).

\subsection{Repeatability}

Thirteen subjects were randomly selected from the study's total sample. The myocardial strain was measured independently by two radiologists with more than 5 years of experience in the diagnosis of cardiovascular diseases. In addition, one observer measured the myocardial strain after 2 weeks. The inter-observer and intra-observer interclass correlation coefficients (ICCs) of the global radial strain (GRS), global circumferential strain (GCS), and global longitudinal strain (GLS) were calculated separately to assess repeatability.

\subsection{Statistical Analysis}

Statistical analysis was performed using SPSS v21.0. The normality of the distributions for all continuous variables was tested using the Shapiro-Wilk test. The normal distribution data were described as the means \pm standard deviation, whereas the non-normally distribution data were expressed as medians (interquartile ranges) and categorical variables were expressed as frequencies (percentages), respectively. Independent sample student's $t$-tests were used to compare two groups of normally distributed variables. One-way analysis of variance was used to compare multiple groups. Furthermore, Bonferroni and Tamhanes were used for post-hoc comparisons between the two groups. Pearson's and Spearman correlation coefficients were implemented for evaluating correlations between continuous variables as appropriate. In addition, multivariable linear regression was further used to analyze the relationship between the parameters and myocardial strain. Finally, repeatability between observers was evaluated with 13 randomly selected patients using ICCs. $p<0.05$ (two-tailed) was considered statistically significant. 


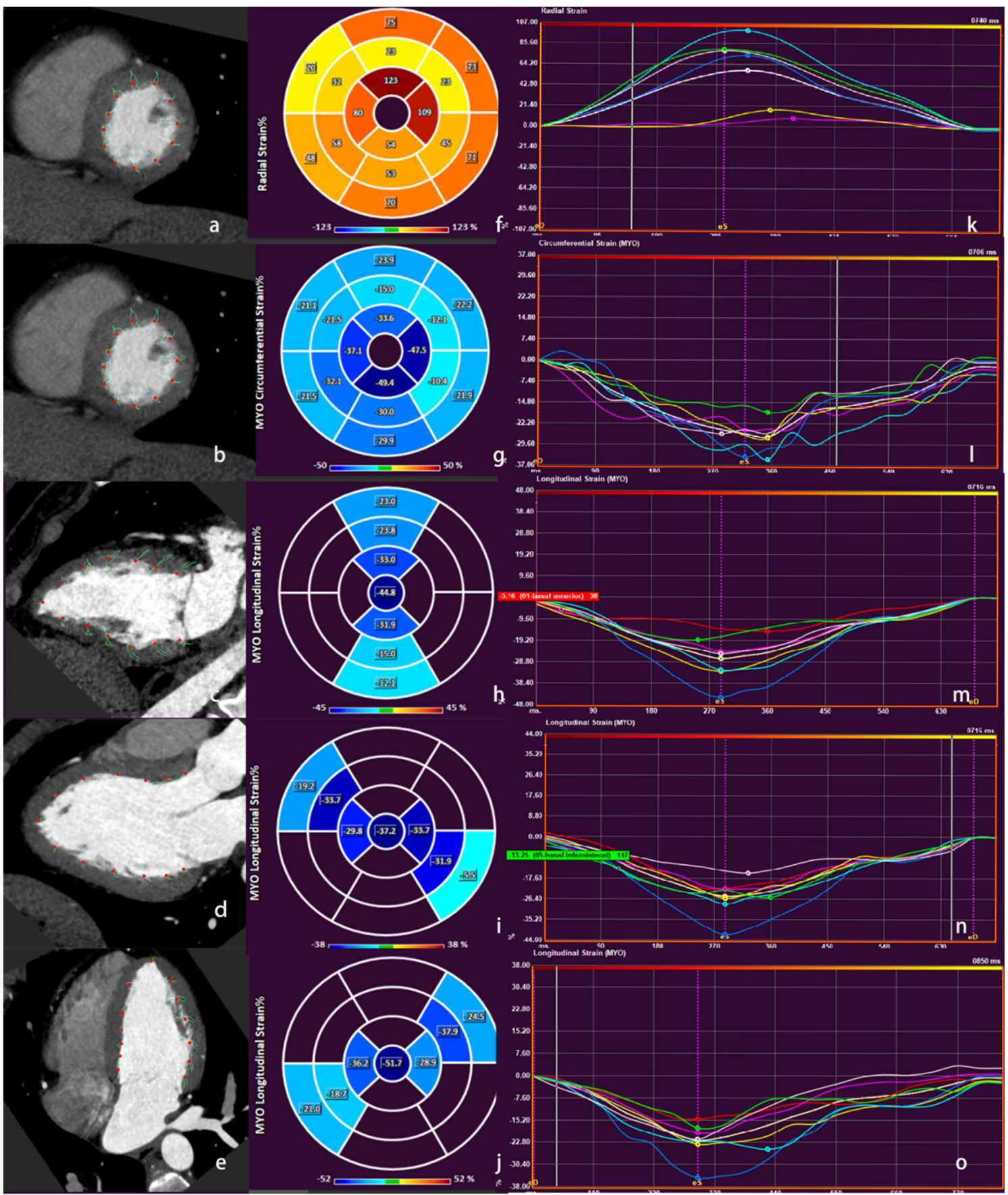

Figure 1. Schematic diagram of myocardial strain on post-processing software. (a-e) Images of different planes of the cardiac; $(\mathbf{f}-\mathbf{j})$ images of Err, Ecc and Ell on the short axis of the cardiac; $(\mathbf{k}-\mathbf{o})$ curves of myocardial strain and time in the cardiac cycle. Err: radial strain; Ecc: circumferential strain; Ell: longitudinal strain.

\section{Results}

\subsection{Clinical Indicators and Cardiac Function Parameters of the Study Population}

In this study, 827 patients with basic clinical information and CCTA examination data were included, and 87 healthy subjects were eventually enrolled (Figure 2), including 41 males and 46 females, aged $27-79$ years ( $48 \pm 11$ years old). The patients were divided into three groups according to age: young ( $\leq 40$ years old), middle-aged ( $40-55$ years old), and elderly ( $>55$ years old). There were statistically significant differences in the age, height, weight, and body mass index (BMI) between males and females, respectively $(p<0.05)$. 
Cardiac function parameters, including LVEDV, LVESV, SV, LVEF, CO, and heart rate (HR), did not differ between the sexes, respectively $(p>0.05)$ (Table 1$)$.

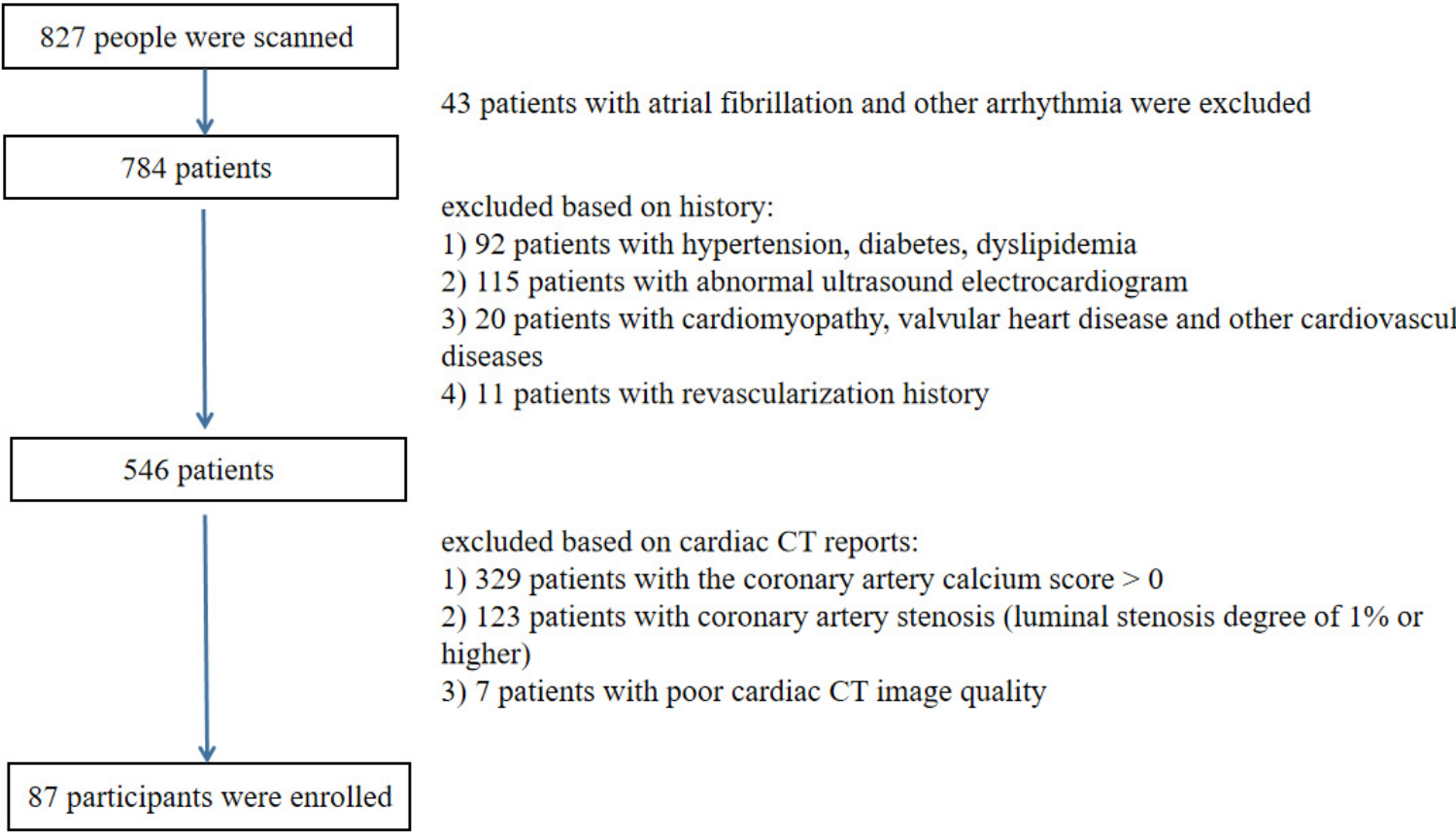

Figure 2. Flowchart of patients who met the inclusion/exclusion criteria for the study.

Table 1. Clinical indicators and cardiac function parameters of the study population.

\begin{tabular}{|c|c|c|c|c|}
\hline Variable & Males $(n=41)$ & Females $(n=46)$ & Total $(n=87)$ & $p$ Values \\
\hline \multicolumn{5}{|l|}{ Age group } \\
\hline$\leq 40$ years old & 16 & 7 & 23 & - \\
\hline $40-55$ years old & 17 & 23 & 40 & - \\
\hline$>55$ years old & 8 & 16 & 24 & - \\
\hline Age (years) & $44.3 \pm 10.9$ & $51.4 \pm 10.3$ & $48.1 \pm 11.1$ & 0.030 * \\
\hline Height $(\mathrm{cm})$ & $169.9 \pm 5.3$ & $160.5 \pm 4.9$ & $165.0 \pm 6.9$ & $<0.001 *$ \\
\hline Weight (kg) & $67.3 \pm 9.2$ & $56.2 \pm 7.1$ & $61.6 \pm 9.9$ & $0.001 *$ \\
\hline $\mathrm{BMI}\left(\mathrm{kg} / \mathrm{m}^{2}\right)$ & $23.3 \pm 2.6$ & $21.8 \pm 2.7$ & $22.5 \pm 2.7$ & $0.014^{*}$ \\
\hline $\mathrm{HR}(($ beats / min) & $66.7 \pm 11.3$ & $70.6 \pm 13.1$ & $68.7 \pm 12.3$ & 0.155 \\
\hline \multicolumn{5}{|c|}{ Cardiac function parameters } \\
\hline LVEDV (mL) & $71.0 \pm 14.6$ & $65.7 \pm 9.9$ & $68.2 \pm 12.5$ & 0.050 \\
\hline LVESV (mL) & $30.5 \pm 9.6$ & $27.7 \pm 6.7$ & $29.0 \pm 8.3$ & 0.125 \\
\hline $\mathrm{SV}(\mathrm{mL})$ & $40.8 \pm 8.7$ & $37.9 \pm 5.8$ & $39.2 \pm 7.4$ & 0.084 \\
\hline LVEF (\%) & $57.7 \pm 6.9$ & $58.1 \pm 6.5$ & $57.9 \pm 6.6$ & 0.752 \\
\hline $\mathrm{CO}(\mathrm{L} / \mathrm{min})$ & $2.8 \pm 0.7$ & $2.7 \pm 0.6$ & $2.7 \pm 0.6$ & 0.643 \\
\hline
\end{tabular}

\subsection{Global and Regional Myocardial Strain of the Left Ventricle}

In this study, 4176 strain values of myocardial segments were measured. After excluding segments with poor image quality and abnormal local myocardial strain due to severe myocardial bridge, 4134 values were statistically analyzed. The global and segment myocardial strain values of the left ventricle are shown in Figure 3.

The myocardial strain in different parts of the left ventricle is shown in Table 2. The Err of the middle segment of the left ventricle was lower than that of the basal and apex segments. I Ecc I (the absolute value of Ecc) in the apical segment was higher than that in the basal and middle segments, and the base segment was higher than that in the 
middle segment. I Ell | (the absolute value of Ell) gradually increased from the basal to the apex segment.
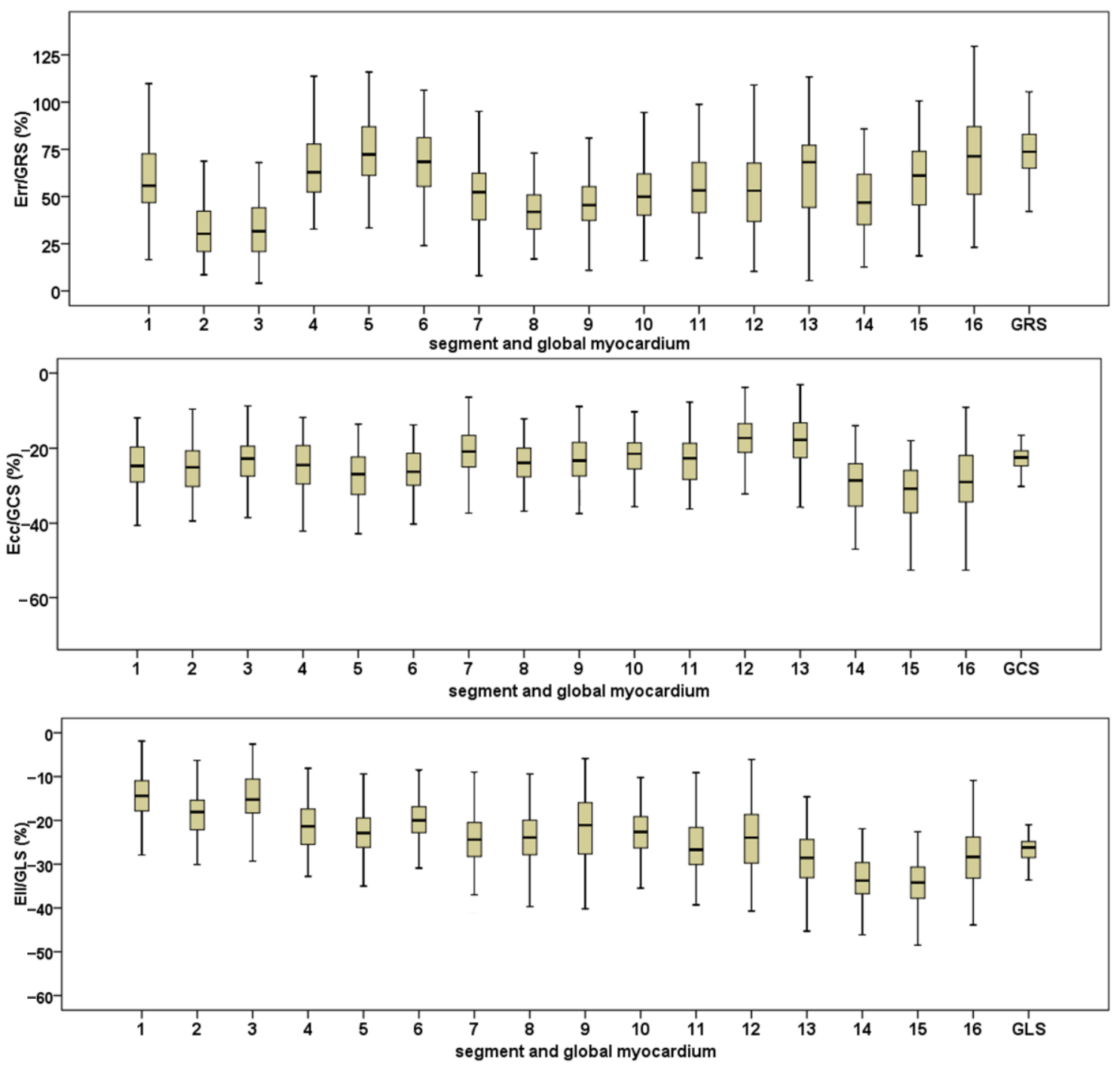

Figure 3. The boxplots for the global and segment myocardial strain value. The top is the image of about 16 segments and the global radial strain. The middle is the image of about 16 segments and the global circumferential strain. The bottom is the image of about 16 segments and the global longitudinal strain. The center box of the boxplots indicates the values from the lower quartile to the higher quartile. The center line shows the median. The range of whiskers is from the minimum to the maximum value. GRS: global radial strain; GCS: global circumferential strain; GLS: global longitudinal strain. Err: radial strain; Ecc: circumferential strain; Ell: longitudinal strain.

Table 2. Myocardial strain in different parts of the left ventricle.

\begin{tabular}{ccccc}
\hline & The Basal & The Middle & The Apical & $p$ Values \\
\hline Err (\%) & $57.0 \pm 12.2$ & $51.2 \pm 15.7$ & $62.7 \pm 22.1^{\mathrm{b}}$ & $<0.001$ \\
Ecc (\%) & $-24.9 \pm 3.6$ & $-21.8 \pm 3.0^{\mathrm{a}}$ & $-27.4 \pm 5.9^{\mathrm{a}, \mathrm{b}}$ & $<0.001$ \\
Ell $(\%)$ & $-18.6 \pm 2.8$ & $-23.8 \pm 3.5^{\mathrm{a}}$ & $-31.6 \pm 4.0^{\mathrm{a}, \mathrm{b}}$ & $<0.001$ \\
\hline
\end{tabular}

Note: All data are expressed as the mean \pm SD. Err: radial strain; Ecc: circumferential strain; Ell: longitudinal strain. ${ }^{\mathrm{a}}$ : $p<0.0167$ compared with the basal segment; ${ }^{\mathrm{b}}: p<0.0167$ compared with the middle segment. 
The myocardial strain in different ventricular walls of the left ventricle is shown in Table 3. In the lateral wall of the left ventricle, Err and | Ell | were the largest, and | Ecc | was the smallest. Err showed as the lateral wall $>$ inferior wall $>$ anterior wall $>$ septal wall. $\mid$ Ecc $\mid$ showed as the inferior wall $>$ septal wall $>$ anterior wall $>$ lateral wall. | Ell $\mid$ showed a clockwise decreasing trend from the lateral wall in the short axis (Table 3).

Table 3. Myocardial strain in different ventricular walls of the left ventricle.

\begin{tabular}{cccccc}
\hline & Anterior & Septal & Inferior & Lateral & $p$ Values \\
\hline Err $(\%)$ & $54.0 \pm 12.2$ & $41.2 \pm 9.2^{\mathrm{a}}$ & $56.3 \pm 10.6^{\mathrm{b}}$ & $66.1 \pm 15.8^{\mathrm{a}, \mathrm{b}, \mathrm{c}}$ & $<0.001$ \\
Ecc $(\%)$ & $-24.9 \pm 3.6$ & $-24.9 \pm 3.8$ & $-27.4 \pm 5.9^{\mathrm{a}, \mathrm{b}}$ & $-24.4 \pm 4^{\mathrm{c}}$ & $<0.001$ \\
Ell $(\%)$ & $-22.1 \pm 3.5$ & $-22.4 \pm 4.0$ & $-23.3 \pm 3.6$ & $-24.5 \pm 4.3^{\mathrm{a}, \mathrm{b}}$ & $<0.001$ \\
\hline
\end{tabular}

Note: All data are expressed as the mean \pm SD. Err: radial strain; Ecc: circumferential strain; Ell: longitudinal strain. ${ }^{\mathrm{a}}: p<0.008$ compared with anterior wall; ${ }^{\mathrm{b}}: p<0.008$ compared with septal wall; ${ }^{\mathrm{c}}: p<0.008$ compared with inferior wall.

\subsection{The Relationship between Left Ventricular Myocardial Strain and Clinical Characteristics}

There were differences in the left ventricular GRS, GLS, and GCS based on the gender of the participant (Table 4). The GRS, I GCS I , and IGLS I (the absolute values of GCS and GLS) in females were higher than those in males (GRS: (77.7 \pm 14.7$) \%$ vs. $(70.9 \pm 15.1) \%$, $p<0.05$; GLS: $(-27.3 \pm 2.6) \%$ vs. $(-25.8 \pm 3.6) \%, p<0.05 ;$ GCS: $(-23.0 \pm 3.1) \%$ vs. $(-22.3 \pm 2.9) \%, p=0.286)$. The differences in myocardial strain on segment and region of the left ventricle in gender are shown in Figure 4.

Table 4. Global myocardial strain of the left ventricle in different genders.

\begin{tabular}{cccc}
\hline & Males & Females & $p$ Values \\
\hline GRS (\%) & $70.9 \pm 15.1$ & $77.7 \pm 14.7$ & $0.038 *$ \\
GCS (\%) & $-22.3 \pm 2.9$ & $-23.0 \pm 3.1$ & 0.286 \\
GLS (\%) & $-25.8 \pm 3.6$ & $-27.3 \pm 2.6$ & $0.023 *$
\end{tabular}

Note: All data are expressed as the mean \pm SD. GRS: global radial strain; GCS: global circumferential strain; GLS: global longitudinal strain. ${ }^{*} p<0.05$.

The correlation between left ventricular myocardial strains, age, and BMI is shown in Figure 5. GRS was significantly positively correlated with age $(\mathrm{r}=0.219, p<0.05)$. While GCS and GLS were negatively correlated with age respectively $(\mathrm{r}=-0.127, p=0.242$; $\mathrm{r}=-0.209, p=0.052)$. GCS and GLS were significantly positively correlated with BMI respectively $(\mathrm{r}=0.274, p<0.05 ; \mathrm{r}=0.223, p<0.05)$. In addition, LVEDV and LVESV were negatively correlated with age $(\mathrm{r}=-0.222, p<0.05 ; \mathrm{r}=-0.274, p<0.05)$, respectively. There was no significant difference in the GRS, GCS, and GLS among all age groups (Figure 6).

\subsection{The Relationship between Left Ventricular Myocardial Strain and Left Ventricular Function Parameters}

The correlation between left ventricular myocardial strains and left ventricular function parameters is shown in Figure 7. The GRS of the left ventricle was significantly negatively correlated with LVESV $(\mathrm{r}=-0.256, p<0.05)$ and significantly positively correlated with LVEF $(r=0.632, p<0.01)$. GCS was significantly positively correlated with LVESV $(r=0.459$, $p<0.01)$ and significantly negatively correlated with SV, LVEF, and CO ( $\mathrm{r}=-0.432,-0.831$, and -0.323 , respectively, $p<0.01)$. There was a significantly negative correlation with GLS, $\mathrm{SV}$, and LVEF ( $\mathrm{r}=-0.326$ and -0.416 , respectively, $p<0.01$ in each). 

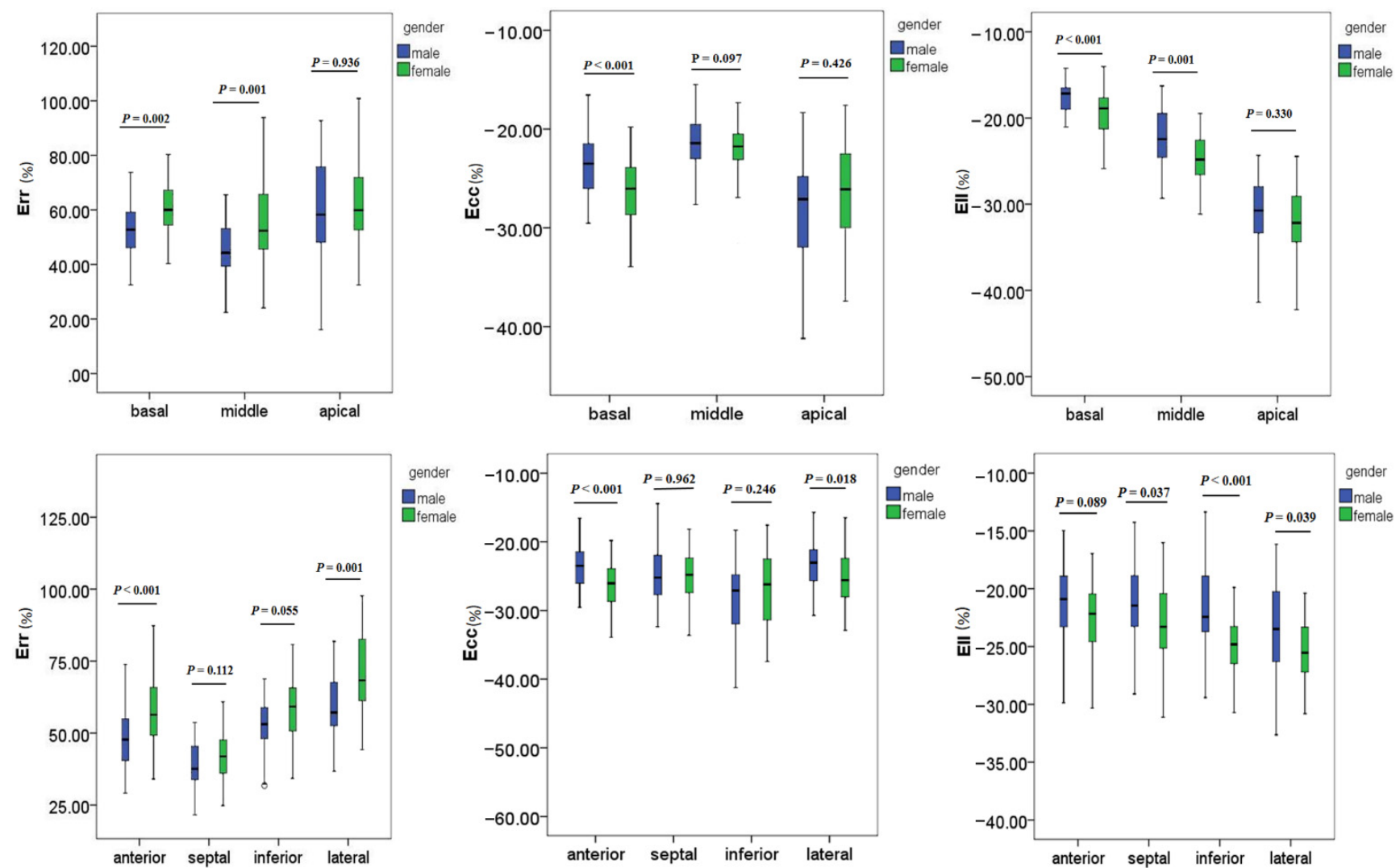

Figure 4. The boxplots for myocardial strain on the level and region of the left ventricular in different genders. The center box of the boxplots indicates the values from the lower quartile to the higher quartile. The center line shows the median. The range of whiskers is from the minimum to the maximum value. Err: radial strain; Ecc: circumferential strain; Ell: longitudinal strain.
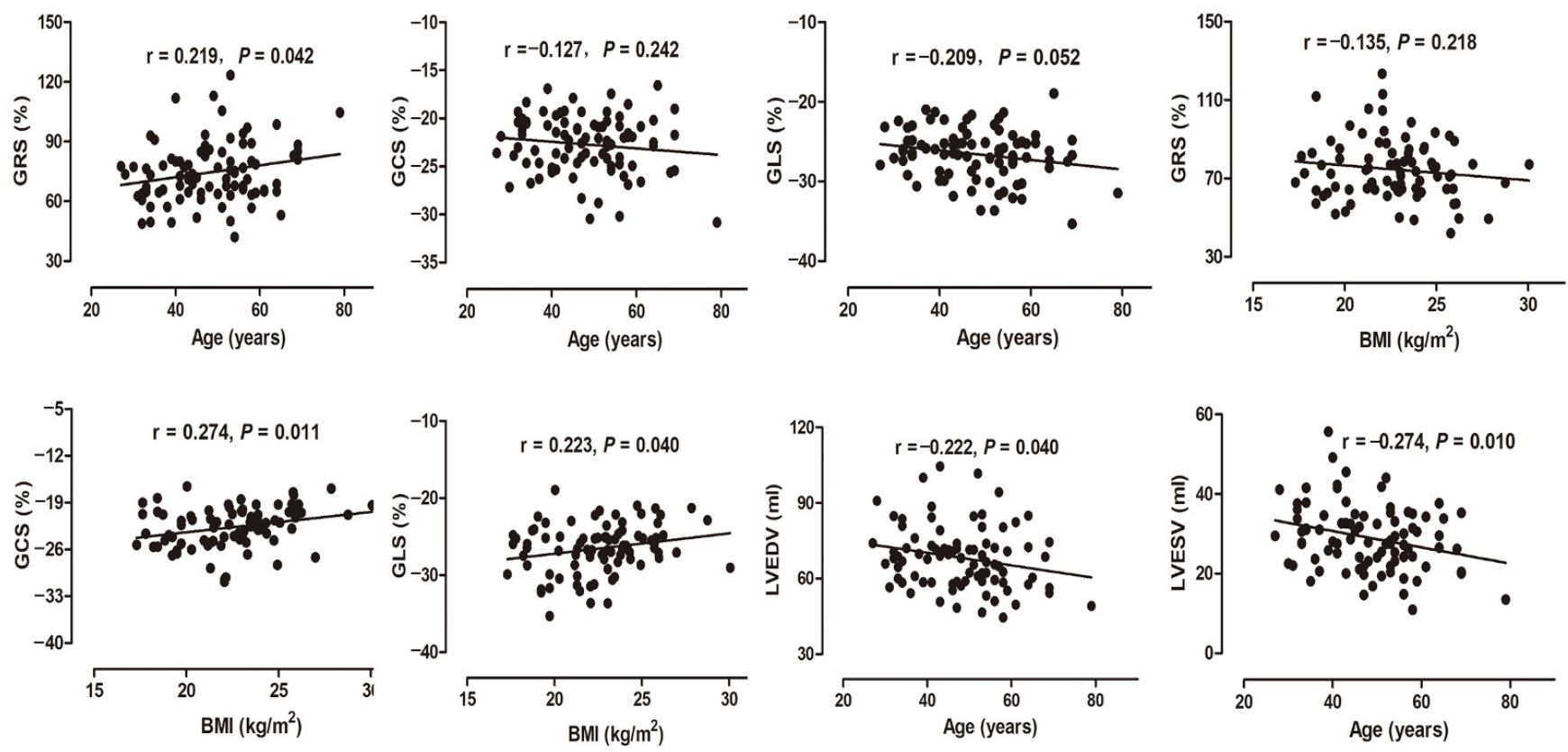

Figure 5. The correlation between left ventricular strain with age and BMI, and the correlation between LVEDV and LVESV with age. GRS: global radial strain; GCS: global circumferential strain; GLS: global longitudinal strain. LVEDV: left ventricular end-diastolic volume; LVESV: left ventricular end-systolic volume; BMI: body mass index. 


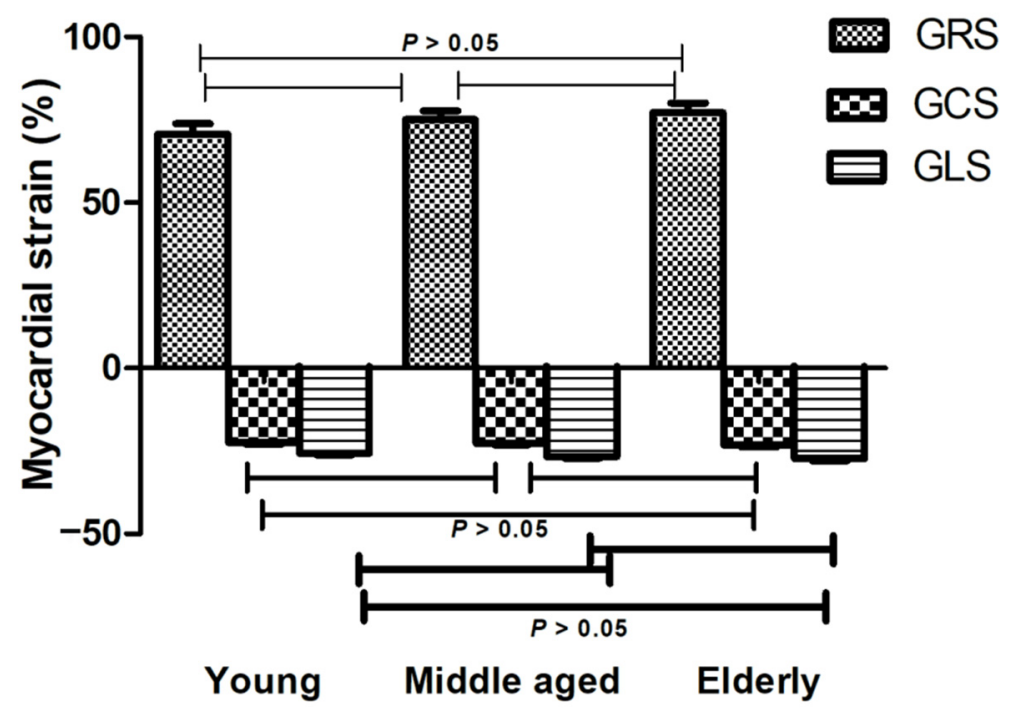

Figure 6. Myocardial strain in different age groups. There was no significant difference in global myocardial strain among all age groups. GRS: global radial strain; GCS: global circumferential strain; GLS: global longitudinal strain.
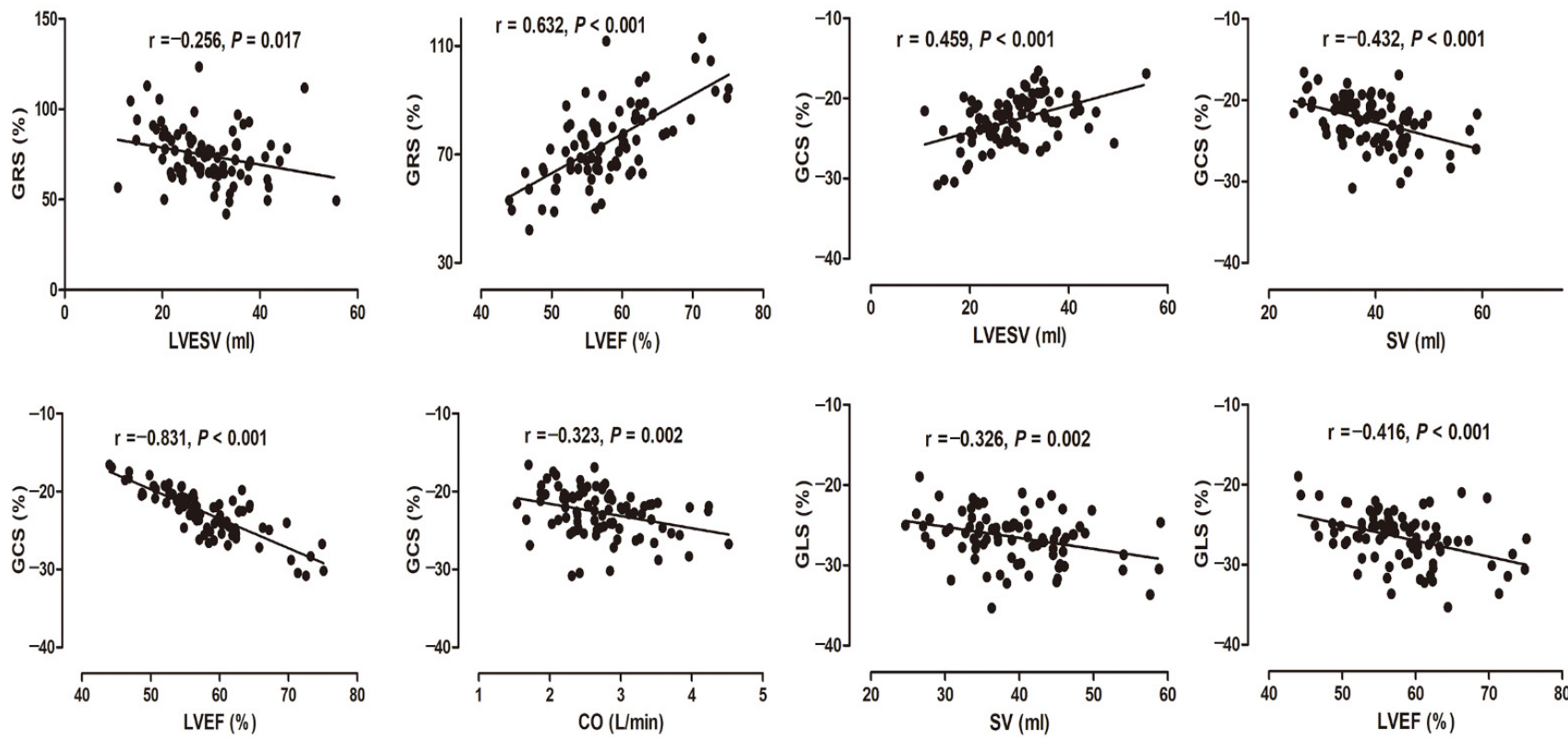

Figure 7. The correlation analysis between left ventricular global myocardial strain and left ventricular function parameters. GRS: global radial strain; GCS: global circumferential strain; GLS: global longitudinal strain. LVEDV: left ventricular end-diastolic volume; LVESV: left ventricular end-systolic volume; SV: stroke volume; CO: cardiac output; LVEF: left ventricular ejection fraction.

Furthermore, univariate and multivariate regression analysis for global myocardial strains and clinical indicators and cardiac function parameters are shown in Table 5. Both the SV (GRS: $\beta=0.481, p=0.004$; GCS: $\beta=-0.115, p<0.001$; GLS: $\beta=-0.14, p=0.003$ ) and LVEF (GRS: $\beta=1.179, p<0.001$; GCS: $\beta=-0.345, p<0.001$; GLS: $\beta=-0.13, p=0.01$ ) were the independent determinants of GRS, GCS, and GLS. The GRS of females was $8.174 \%$ higher than males, and the GLS was $-1.653 \%$ lower than males. BMI $(\beta=0.255, p<0.001)$ and $\mathrm{CO}(\beta=0.854, p=0.022)$ were the independent determined factors of GCS. 
Table 5. Univariate and multivariate regression analysis for global myocardial strains and clinical indicators and cardiac function parameters.

\begin{tabular}{|c|c|c|c|c|c|c|}
\hline \multirow[t]{2}{*}{ Variable } & \multicolumn{2}{|c|}{$\begin{array}{c}\text { GRS } \\
\text { Univariate Analysis }\end{array}$} & \multicolumn{2}{|c|}{$\begin{array}{c}\text { GCS } \\
\text { Univariate Analysis }\end{array}$} & \multicolumn{2}{|c|}{$\begin{array}{c}\text { GLS } \\
\text { Univariate Analysis }\end{array}$} \\
\hline & $\beta$ Value & $p$ Value & $\beta$ Value & $p$ Value & $\beta$ Value & $p$ Value \\
\hline Age (years) & 0.299 & 0.042 & -0.034 & 0.242 & -0.060 & 0.052 \\
\hline Female * & 6.756 & 0.038 & -0.694 & 0.286 & -1.550 & 0.023 \\
\hline Height (cm) & -0.119 & 0.626 & -0.016 & 0.737 & -0.010 & 0.842 \\
\hline Weight (kg) & -0.208 & 0.427 & 0.057 & 0.088 & 0.050 & 0.160 \\
\hline $\operatorname{BMI}\left(\mathrm{kg} / \mathrm{m}^{2}\right)$ & -0.762 & 0.218 & 0.304 & 0.011 & 0.264 & 0.040 \\
\hline HR (beats/min) & -0.071 & 0.611 & 0.096 & 0.389 & 0.048 & 0.098 \\
\hline LVEDV (mL) & -0.014 & 0.913 & 0.019 & 0.477 & -0.017 & 0.548 \\
\hline LVESV (mL) & -0.472 & 0.016 & 0.167 & $<0.001$ & 0.063 & 0.131 \\
\hline $\mathrm{SV}(\mathrm{mL})$ & 0.738 & $<0.001$ & -0.170 & $<0.001$ & -0.138 & 0.002 \\
\hline LVEF (\%) & 1.448 & $<0.001$ & -0.378 & $<0.001$ & -0.201 & $<0.001$ \\
\hline $\mathrm{CO}(\mathrm{L} / \mathrm{min})$ & 9.282 & $<0.001$ & -1.567 & 0.002 & -0.770 & 0.167 \\
\hline \multirow{2}{*}{ variable } & \multicolumn{2}{|c|}{ Multivariate analysis } & \multicolumn{2}{|c|}{ Multivariate analysis } & \multicolumn{2}{|c|}{ Multivariate analysis } \\
\hline & $\beta$ value & $p$ value & $\beta$ value & $p$ value & $\beta$ value & $p$ value \\
\hline Female * & 8.174 & $<0.001$ & - & - & -1.653 & 0.010 \\
\hline $\operatorname{BMI}\left(\mathrm{kg} / \mathrm{m}^{2}\right)$ & - & - & 0.255 & $<0.001$ & - & - \\
\hline $\mathrm{SV}(\mathrm{mL})$ & 0.481 & 0.004 & -0.115 & $<0.001$ & -0.14 & 0.003 \\
\hline LVEF (\%) & 1.179 & $<0.001$ & -0.345 & $<0.001$ & -0.13 & 0.010 \\
\hline $\mathrm{CO}(\mathrm{L} / \mathrm{min})$ & - & - & 0.854 & 0.022 & - & - \\
\hline
\end{tabular}

Note: * Males as reference; GRS: global radial strain; GCS: global circumferential strain; GLS: global longitudinal strain. BMI: body mass index; HR: heartbeat; LVEDV: left ventricular end-diastolic volume; LVESV: left ventricular end-systolic volume; SV: stroke volume; CO: cardiac output; LVEF: left ventricular ejection fraction. Stepwise linear regression was used to analyze multivariate regression.

\subsection{Reproducibility}

The ICC values in the intra-observer analysis were $0.575,0.900$, and 0.891 for GRS, GCS, and GLS, respectively. Finally, the ICCs were $0.325,0.853$, and 0.787 for GRS, GCS, and GLS, respectively, in the inter-observer analysis (Table 6).

Table 6. The results of reproducibility in global myocardial strains.

\begin{tabular}{ccccc}
\hline & \multicolumn{2}{c}{ Intra-Observer } & \multicolumn{2}{c}{ Inter-Observer } \\
& ICCs & CV & ICCs & CV \\
\hline GRS & 0.575 & $5.0 \%$ & 0.325 & $1.9 \%$ \\
GCS & 0.900 & $0.2 \%$ & 0.853 & $2.1 \%$ \\
GLS & 0.891 & $1.2 \%$ & 0.787 & $1.4 \%$ \\
\hline
\end{tabular}

Notes: GRS: global radial strain; GCS: global circumferential strain; GLS: global longitudinal strain; ICC: intraclass correlation coefficients; CV: coefficient of variation.

\section{Discussion}

It is important to understand the normal global structural and functional parameters of the left ventricle, which is the basis for evaluating cardiovascular diseases. Furthermore, it is worth applying a measurement technique in the research of reference values, as this helps the timely clinical discovery of abnormalities, diagnosis, and treatment.

The normal values of the left ventricular myocardial strains quantified by FT-CT reveal significant differences in gender and segments and correlate with clinical characteristics and left ventricular function. This study shows that the GRS, GCS, and CLS of the left ventricle were $74.5 \pm 15.2 \%,-22.7 \pm 3.01 \%$, and $-26.6 \pm 3.2 \%$, respectively, in healthy individuals. The absolute values measured by FT-CT were higher than the normal values measured by Cao [11] and Liu [4] using the Cvi and Trufi Strain software, respectively. The differences might be related to the scanning equipment and implemented measurement methods. It has been proven that there are differences in measurement methods among different software [2]. The myocardial FT technique is based on identifying features in images and tracking them in continuous images [12], depending on the quality of imaging 
and sharpness of the endocardial boundary. CT examination has high spatial and contrast resolution, and less influence on the myocardium border than CMR, which is caused by blood flow artifacts on the left ventricle. These may be the reasons why the values measured by FT-CT were higher than those measured by FT-CMR.

Our study shows that there were differences in the three short axes of the left ventricular regional myocardial strains. The Err and Ecc of the left ventricle were the highest in the apex and lowest in the middle, which agrees with the results of Ecc measured by Taylor [10] using FT-CMR. However, a different result has also been reported, which showed that Ecc was highest in the apex and lowest in the basal measured by ultrasonic speckle-tracking imaging [13]. This may result from the imaging technology. In contrast, the absolute value of Ell, showing a gradually increasing trend from the base, agrees with the results reported by Leitman [13] and Qian [9].

In addition, significant differences in regional myocardial strains were observed among different segments and walls of the left ventricle, which agrees with previous studies [14-16]. This is the expression of local non-uniformity of the left ventricular function in healthy people, showing differences in myocardial strain among different short axis levels and ventricular walls. The literature suggests that the left ventricle has a high regional morphological and functional non-uniformity in healthy individuals, including transmural, apex to base, and circumferential non-uniformity. This shows heterogeneity in the synchronous contraction of the heart and may impact the effectiveness of regional functional assessments $[17,18]$. The segmental radial strains vary greatly, and the segmental circumferential and longitudinal strains show a relatively consistent normal range. Therefore, it is necessary to quantify the segmental specific reference value for the circumferential and longitudinal strains in healthy individuals.

It has been reported that left ventricular myocardial strains partly differ between genders and age groups in healthy subjects $[3,10,19]$. The study found that the GRS and GLS values of healthy females were higher than those of males, which disagrees with the study by Andre et al. [3], which found that the GRS in women is lower than that in men. In their study, there was a significant difference in the circumferential strain between genders, whereas only one trend was shown in our sample, which may be related to the relatively small sample size, population age, and BMI in our study. However, the differences in the GRS and GCS between genders in our study agree with those of other studies [10,15]. First, there were differences in the volume, mass, and the LVEF of the left ventricle, which is normal between males and females, and differences in myocardial deformation [20,21]. In this study, the GRS was positively correlated with age, and the age of female subjects was greater than that of the male subjects. Furthermore, the GLS was positively correlated with BMI, and the BMI of male samples was greater than that of female subjects. Therefore, the GRS and GLS values of healthy females in the study were higher than those of males. The analysis of the differences in myocardial systolic peak strain between genders can help further research of cardiac movement.

In addition, this study found a significant positive correlation between GRS and age, and a negative correlation between LVEDV, LVESV, and age. The literature shows that with increasing age, LVEDV shrinks significantly and myocardial mass decreases, leading to a significant increase in the left ventricular (LV) mass/LVEDV ratio. Then, LV remodeling occurs, with an increase in myocardial stiffness and a decrease in compliance and diastolic function, which results in a compensatory thickening of the LV myocardium during systole, eventually leading to the increase of GRS [22]. Meanwhile, the study showed a significant positive correlation between GRS and LVEF, and a significant negative correlation between GCS, GLS, and LVEF. An increase in myocardial strain indicates stretching of the LV myocardial fibers, myocardial thickening, and increased myocardial contractility, with a corresponding increase in LVEF. Conversely, a decrease in myocardial strain indicates the shortening of myocardial fibers, myocardial thinning, and a corresponding decrease in LVEF [18]. Thus, it may provide incremental diagnostic value when studying the ominous 
decrease in ejection fraction after systolic heart failure or myocardial infarction using a comprehensive assessment of LV systolic function with myocardial strain.

Another significant positive correlation was revealed between left ventricular GCS, GLS, and BMI in healthy individuals, and similar results have not been reported in the literature. Whether GCS and GLS based on cardiac CT can become early warning indicators to quantify the health status of obese people remains to be studied.

In this study, GCS and GLS had good intra-observer and inter-observer reproducibility, and GRS measurements were less stable. The poor reproducibility of the radial parameters may be caused by the quantification of the radial strain, which depends on the simultaneous motion of the endocardium and epicardium. Furthermore, the density contrast at the epicardial border is less pronounced than at the endocardial border. Moreover, there are more myocardial trabeculae in the apical region. The compression and drainage of blood from the trabeculae at the end of systole alter the voxel appearance in this region, making accurate tracking challenging [19]. Finally, when the blood space between the trabeculae closes during systole, the border between the trabeculae and the dense portion of the myocardium may move, resulting in blurring of the endocardial contours.

This study has some limitations. First, it is a single-center, single-race study. Therefore, the sample size of males and females were unequal, and there is a possibility that some gender differences were magnified or inadequately detected. Considering the limited overall sample size and the number of samples from youth and elderly groups, the results of myocardial strain between groups stratified by age may require further analysis by expanding the sample size. Second, this study did not perform a concordance test between myocardial strain measured using CT FT techniques and other imaging techniques. However, previous studies have shown good concordance between CT FT techniques, ultrasound speckle-tracking techniques, and FT-CMR to assess myocardial strain in patients with cardiovascular disease. Lastly, the radiation dose was relatively high due to the use of a retrospective electrocardiogram gated scan. However, we used techniques (CarekV and CareDose) to reduce the radiation dose and ensure a one-stop evaluation of coronary anatomy and myocardial strains at a comparable dose. In addition, compared with the radiation dose described by the authors of [23] and the diagnostic reference level of an international cardiac CT [24] (DLP was $348 \mathrm{mGy} \cdot \mathrm{cm}, 400 \mathrm{mGy} \cdot \mathrm{cm}$, respectively), our average effective radiation dose was reasonable and was not higher than theirs (average effective radiation dose: $4.0 \pm 1.4 \mathrm{mSv}$ ).

\section{Conclusions}

Conclusively, using the CT FT technique to assess left ventricular myocardial strain in healthy individuals is feasible and reproducible. In the future, the overall sample size can be increased to quantify the reference values of LV myocardial strain in different age groups. Furthermore, given the rapidity and convenience of CT examination, using CT to quantitatively assess myocardial strain differences by gender and segment should improve the quantitative diagnosis of cardiovascular diseases, such as coronary heart disease and cardiomyopathy.

Author Contributions: Conceptualization, N.L. and T.L.; methodology, N.L. and J.L.; software, Y.C. and J.Y.; validation, Y.L. and M.Y.; formal analysis, N.L. and T.L.; investigation, W.Z. and H.S.; resources, W.Z. and H.S.; data curation, H.S.; writing—original draft preparation, N.L.; writing—review and editing, Z.X. and H.S.; visualization, G.S. and X.H.; supervision, Y.C.; project administration, H.S. All authors have read and agreed to the published version of the manuscript.

Funding: This research was supported by the National Natural Science Foundation of China under Grant 81271570.

Institutional Review Board Statement: The study was conducted according to the guidelines of the Declaration of Helsinki, and approved by the Institutional Review Board of Tongji Medical College, Huazhong University of Science and Technology (S 878; approval date on May 2019).

Informed Consent Statement: Patient consent was waived because this is a retrospective study. 
Data Availability Statement: The data presented in this study are available on request from the corresponding author.

Conflicts of Interest: The authors declare no conflict of interest.

\section{References}

1. Scatteia, A.; Baritussio, A.; Bucciarelli-Ducci, C. Strain imaging using cardiac magnetic resonance. Hear. Fail. Rev. 2017, 22, 465-476. [CrossRef] [PubMed]

2. Bucius, P.; Erley, J.; Tanacli, R.; Zieschang, V.; Giusca, S.; Korosoglou, G.; Steen, H.; Stehning, C.; Pieske, B.; Pieske-Kraigher, E.; et al. Comparison of feature tracking, fast-SENC, and myocardial tagging for global and segmental left ventricular strain. ESC Heart Fail. 2020, 7, 523-532. [CrossRef] [PubMed]

3. Andre, F.; Steen, H.; Matheis, P.; Westkott, M.; Breuninger, K.; Sander, Y.; Kammerer, R.; Galuschky, C.; Giannitsis, E.; Korosoglou, G.; et al. Age- and gender-related normal left ventricular deformation assessed by cardiovascular magnetic resonance feature tracking. J. Cardiovasc. Magn. Reson. 2015, 17, 25. [CrossRef] [PubMed]

4. Liu, H.; Yang, D.; Wan, K.; Luo, Y.; Sun, J.-Y.; Zhang, T.-J.; Li, W.-H.; Greiser, A.; Jolly, M.-P.; Zhang, Q.; et al. Distribution pattern of left-ventricular myocardial strain analyzed by a cine MRI based deformation registration algorithm in healthy Chinese volunteers. Sci. Rep. 2017, 7, 45314. [CrossRef]

5. Ammon, F.; Bittner, D.; Hell, M.; Mansour, H.; Achenbach, S.; Arnold, M.; Marwan, M. CT-derived left ventricular global strain: A head-to-head comparison with speckle tracking echocardiography. Int. J. Cardiovasc. Imaging 2019, 35, 1701-1707. [CrossRef]

6. Miskinyte, E.; Bucius, P.; Erley, J.; Zamani, S.M.; Tanacli, R.; Stehning, C.; Schneeweis, C.; Lapinskas, T.; Pieske, B.; Falk, V.; et al. Assessment of Global Longitudinal and Circumferential Strain Using Computed Tomography Feature Tracking: Intra-Individual Comparison with CMR Feature Tracking and Myocardial Tagging in Patients with Severe Aortic Stenosis. J. Clin. Med. 2019, 8, 1423. [CrossRef]

7. Szilveszter, B.; Nagy, A.I.; Vattay, B.; Apor, A.; Kolossváry, M.; Bartykowszki, A.; Simon, J.; Drobni, Z.D.; Tóth, A.; Suhai, F.I.; et al. Left ventricular and atrial strain imaging with cardiac computed tomography: Validation against echocardiography. J. Cardiovasc. Comput. Tomogr. 2020, 14, 363-369. [CrossRef]

8. Liu, H.; Yang, D.; Lu, Y.; Wan, K.; Wang, S.; Zhang, T.; Li, W.; Zhang, Q.; Chen, Y.; Sun, J. Reference Values for Left Ventricular Myocardial Strains Measured by Feature-tracking Magnetic Resonance Imaging in Chinese Han Population. J. Sichuan Univ. 2016, 47, 599-604.

9. Qian, S.; Yang, L.; Gao, H.; Li, Y.; Zhang, J.; Qiao, W. Three different speckle-tracking techniques in assessment of leftventricular myocardial strains in healthy subjects. Chin. J. Med. Imaging Technol. 2015, 31, 854-858.

10. Taylor, R.; Moody, W.E.; Umar, F.; Edwards, N.C.; Taylor, T.J.; Stegemann, B.; Townend, J.; Hor, K.N.; Steeds, R.; Mazur, W.; et al. Myocardial strain measurement with feature-tracking cardiovascular magnetic resonance: Normal values. Eur. Heart J. Cardiovasc. Imaging 2015, 16, 871-881. [CrossRef]

11. Cao, Y.; Cui, Y.; Zeng, W.; Kong, X.; Zhang, S.; Song, J.; Shi, H. Quantification of myocardial tissue characterization andstrain of the left ventricle in healthy volunteers with 1.5T magnetic resonance: A preliminary study. J. Clin. Cardiol. 2017, 11, 1077-1082.

12. Pedrizzetti, G.; Claus, P.; Kilner, P.J.; Nagel, E. Principles of cardiovascular magnetic resonance feature tracking and echocardiographic speckle tracking for informed clinical use. J. Cardiovasc. Magn. Reason. 2016, 18, 51. [CrossRef] [PubMed]

13. Leitman, M.; Lysiansky, M.; Lysyansky, P.; Friedman, Z.; Tyomkin, V.; Fuchs, T.; Adam, D.; Krakover, R.; Vered, Z. Circumferential and Longitudinal Strain in 3 Myocardial Layers in Normal Subjects and in Patients with Regional Left Ventricular Dysfunction. J. Am. Soc. Echocardiogr. 2010, 23, 64-70. [CrossRef] [PubMed]

14. Kleijn, S.A.; Pandian, N.G.; Thomas, J.D.; De Isla, L.P.; Kamp, O.; Zuber, M.; Nihoyannopoulos, P.; Forster, T.; Nesser, H.-J.; Geibel, A.; et al. Normal reference values of left ventricular strain using three-dimensional speckle tracking echocardiography: Results from a multicentre study. Eur. Heart J. Cardiovasc. Imaging 2015, 16, 410-416. [CrossRef]

15. Muraru, D.; Cucchini, U.; Mihăilă, S.; Miglioranza, M.H.; Aruta, P.; Cavalli, G.; Cecchetto, A.; Padayattil-Josè, S.; Peluso, D.; Iliceto, S.; et al. Left Ventricular Myocardial Strain by Three-Dimensional Speckle-Tracking Echocardiography in Healthy Subjects: Reference Values and Analysis of Their Physiologic and Technical Determinants. J. Am. Soc. Echocardiogr. 2014, $27,858-871$. [CrossRef]

16. Maffessanti, F.; Nesser, H.-J.; Weinert, L.; Steringer-Mascherbauer, R.; Niel, J.; Gorissen, W.; Sugeng, L.; Lang, R.M.; Mor-Avi, V. Quantitative Evaluation of Regional Left Ventricular Function Using Three-Dimensional Speckle Tracking Echocardiography in Patients with and Without Heart Disease. Am. J. Cardiol. 2009, 104, 1755-1762. [CrossRef]

17. Kleijn, S.A.; Aly, M.F.A.; Terwee, C.B.; van Rossum, A.C.; Kamp, O. Three-Dimensional Speckle Tracking Echocardiography for Automatic Assessment of Global and Regional Left Ventricular Function Based on Area Strain. J. Am. Soc. Echocardiogr. 2011, 24, 314-321. [CrossRef]

18. Bogaert, J.; Rademakers, F.E. Regional nonuniformity of normal adult human left ventricle. Am. J. Physiol. Heart Circ. Physiol. 2001, 280, H610-H620. [CrossRef]

19. Augustine, D.; Lewandowski, A.J.; Lazdam, M.; Rai, A.; Francis, J.; Myerson, S.; Noble, A.; Becher, H.; Neubauer, S.; Petersen, S.E.; et al. Global and regional left ventricular myocardial deformation measures by magnetic resonance feature tracking in healthy volunteers: Comparison with tagging and relevance of gender. J. Cardiovasc. Magn. Reson. 2013, 15, 8. [CrossRef] 
20. Natori, S.; Lai, S.; Finn, J.P.; Gomes, A.S.; Hundley, W.G.; Jerosch-Herold, M.; Pearson, G.; Sinha, S.; Arai, A.; Lima, J.A.C.; et al. Cardiovascular Function in MultiEthnic Study of Atherosclerosis: Normal Values by Age, Sex, and Ethnicity. Am. J. Roentgenol. 2006, 186, S357-S365. [CrossRef]

21. Le, T.-T.; Tan, R.S.; De Deyn, M.; Goh, E.P.C.; Han, Y.; Leong, B.R.; Cook, S.A.; Chin, C.W.-L. Cardiovascular magnetic resonance reference ranges for the heart and aorta in Chinese at 3T. J. Cardiovasc. Magn. Reson. 2016, 18, 21. [CrossRef] [PubMed]

22. Cheng, S.; Fernandes, V.R.; Bluemke, D.A.; McClelland, R.L.; Kronmal, R.A.; Lima, J.A. Age-Related Left Ventricular Remodeling and Associated Risk for Cardiovascular Outcomes. Circ. Cardiovasc. Imaging 2009, 2, 191-198. [CrossRef]

23. Yoshida, K.; Tanabe, Y.; Kido, T.; Kurata, A.; Uraoka, D.; Kinoshita, M.; Uetani, T.; Nishimura, K.; Inoue, K.; Ikeda, S.; et al. Characteristics of the left ventricular three-dimensional maximum principal strain using cardiac computed tomography: Reference values from subjects with normal cardiac function. Eur. Radiol. 2020, 30, 6109-6117. [CrossRef] [PubMed]

24. Stocker, T.J.; Deseive, S.; Leipsic, J.; Hadamitzky, M.; Chen, M.Y.; Rubinshtein, R.; Heckner, M.; Bax, J.J.; Fang, X.-M.; Grove, E.L.; et al. Reduction in radiation exposure in cardiovascular computed tomography imaging: Results from the prospective multicenter registry on radiation dose Estimates of cardiac CT angiography in daily practice in 2017 (PROTECTION VI). Eur. Heart J. 2018, 39, 3715-3723. [CrossRef] [PubMed] 